Article

\title{
Pore Structures for High-Throughput Nanopore Devices
}

\author{
Sou Ryuzaki ${ }^{1,2, * \mathbb{D}}$, Rintaro Matsuda ${ }^{1}$ and Masateru Taniguchi ${ }^{3, *}$ \\ 1 Institute for Materials Chemistry and Engineering, Kyushu University, Fukuoka 819-0395, Japan; \\ matsuda@ms.ifoc.kyushu-u.ac.jp \\ PRESTO, Japan Science and Technology Agency (JST), Saitama 332-0012, Japan \\ 3 The Institute of Scientific and Industrial Research, Osaka University, Osaka 567-0047, Japan \\ * Correspondence: ryuzaki@ms.ifoc.kyushu-u.ac.jp (S.R.); taniguti@sanken.osaka-u.ac.jp (M.T.); \\ Tel.: +81-092-642-2726 (S.R.); +81-6-6875-2440 (M.T.)
}

Received: 23 August 2020; Accepted: 25 September 2020; Published: 26 September 2020

check for updates

\begin{abstract}
Nanopore devices are expected to advance the next-generation of nanobiodevices because of their strong sensing and analyzing capabilities for single molecules and bioparticles. However, the device throughputs are not sufficiently high. Although analytes pass through a nanopore by electrophoresis, the electric field gradient is localized inside and around a nanopore structure. Thus, analytes located far from a nanopore cannot be driven by electrophoresis. Here, we report nanopore structures for high-throughput sensing, namely, inverted pyramid (IP)-shaped nanopore structures. Silicon-based IP-shaped nanopore structures create a homogeneous electric field gradient within a nanopore device, indicating that most of the analytes can pass through a nanopore by electrophoresis, even though the analytes are suspended far from the nanopore entrance. In addition, the nanostructures can be fabricated only by photolithography. The present study suggests a high potential for inverted pyramid shapes to serve as nanopore devices for high-throughput sensing.
\end{abstract}

Keywords: nanopore; high-throughput; electric field; nanofluidics

\section{Introduction}

One of the most extreme nanofluidics in nanobiotechnology is a channel with the size of a single molecule, because these nanochannels enable us to handle and analyze single molecules. For physiological tissues, nanochannels, e.g., membrane transport protein, are also essential systems for the detection and discrimination of single biomolecules in our bodies [1]. Nanopore devices have been a research focus from the nanochannel perspective because the devices are intended to mimic the functions of membrane transport proteins, although these devices are only available for large molecules or bioparticles at the moment [2-4], e.g., DNA sequencers [5] and single-bioparticle analyzers [6].

A nanopore device typically consists of an insulating membrane with a single nanopore structure, which physically separates the cis and trans chambers, and it has an electrode pair across the membrane. The chambers are filled with an electrolyte solution, and analytes dispersed in the cis chamber translocate to the trans chamber via a nanopore by electrophoresis (Figure 1a). Nanopore devices provide size and/or shape information for single analytes passing through a nanopore by probing temporal changes in the ionic current because an analyte excludes ions inside a nanopore during the translocation and causes an ionic current blockade with amplitudes proportional to its feature size $[7,8]$. Nanopore structures thus enable label-free sensing of single biomolecules and bioparticles by estimating the size and/or shape of the analytes passing through a nanopore from the pulsed ionic current blockades. However, the device throughput is not sufficiently high because the potential drop is localized inside and around a nanopore structure (Figure A1). Thus, it is difficult to drive analytes far 
from a nanopore with electrophoresis, meaning that only analytes around the nanopore entrance can be detected unless analytes at a distance greater than the capture radius diffuse into the region incidentally. A large number of publications report how to improve the throughput by optimizing electrolyte concentration [9], controlling the local charge on nanopore walls [10], and increasing the number of nanopore structures in a device, such as an array structure [11-13]. However, the throughput has only increased several times per nanopore structure because the potential drop is basically localized inside and around a nanopore structure. One of the relevant factors for the localized potential drop is a higher pore resistance $\left(R_{\text {pore }}\right)$ compared with access resistances $\left(R_{\text {acc }}\right)$, which corresponds to the ionic current resistances inside a nanopore and between the electrode and the nanopore, respectively. Properties of nanopore devices are often discussed with a simple equivalent circuit consisting of the $R_{\text {pore }}$ and the $R_{\text {acc }}$ in a series (Figure $1 \mathrm{~b}$ ). In this instance, each resistance can be described by

$$
\begin{gathered}
R_{\text {pore }}=\frac{\alpha h}{\pi\left(D_{\mathrm{c}} / 2\right)^{2}} \\
R_{\mathrm{acc}}=\frac{\alpha}{D_{\mathrm{c}}}
\end{gathered}
$$

where $\alpha$ is the resistivity of the buffer solution, $h$ is the thickness of a nanopore, and $D_{\mathrm{c}}$ is the diameter

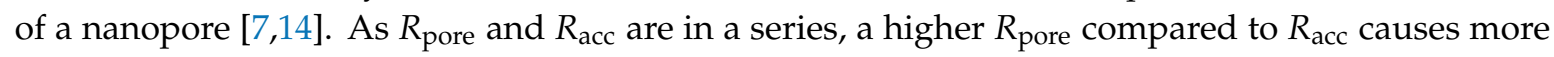
drastic potential drop inside a nanopore. Although a larger $D_{\mathrm{c}}$ can make the ratio of $R_{\text {pore }}$ to $R_{\text {acc }}$ smaller, a larger $D_{c}$ results in a lower signal/noise $(\mathrm{S} / \mathrm{N})$ ratio of the ionic current blockades due to analyte translocations. The insulating membrane is also the factor causing the localized potential drop because the permittivity of the insulating membrane is a relatively small value, and the combined resistance of $R_{\text {pore }}$ and $R_{\mathrm{m}}$, which is $\left(R_{\mathrm{m}} \times R_{\text {pore }}\right) /\left(R_{\mathrm{m}}+R_{\text {pore }}\right)$, is considerably higher than $R_{\mathrm{acc}}$. Here, $R_{\mathrm{m}}$ corresponds to a resistance of the insulating membrane. Therefore, the potential shows a drastic drop inside the membrane and nanopore, resulting in almost flat electric field gradients in the cis and trans chambers, and small gradients in the vicinity of the nanopore entrance and exit (Figure A1). Although homogeneous electric field gradients in each chamber are obtained using a conducting membrane, the noise caused by the membrane in the ionic current increases because of the high capacitance of a conducting membrane $\left(C_{m}\right)[15]$.

(a)



(b)

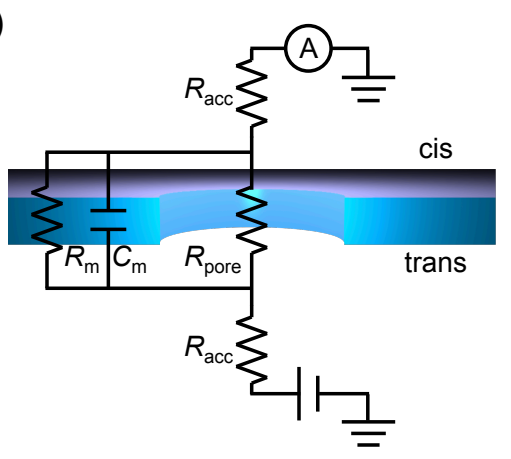

Figure 1. Schematic illustrations of a nanopore device. (a) Schematic illustration of analyte translocations by electrophoresis around a nanopore. As the potential drop is localized inside and around a nanopore, only analytes around the nanopore entrance can be detected. (b) Equivalent circuit of a nanopore device. $R_{\text {ass }}, R_{\text {pore }}, R_{\mathrm{m}}$, and $C_{\mathrm{m}}$ correspond to the access resistance, the pore resistance, the membrane resistance, and the membrane capacitance, respectively.

Here, we report inverted pyramid (IP)-shaped nanopore structures consisting of a layered structure of insulating silicon nitride $\left(\mathrm{Si}_{3} \mathrm{~N}_{4}\right)$ and semiconducting Silicon ( $\mathrm{Si}$ ) membranes to even out the electric field in a nanopore device. This structure is expected to cause a homogeneous potential drop between the electrodes and the nanopore structure because the large pore entrance of the IP shape $(D)$ causes 
a smaller $R_{\text {pore }}$ than that of a normal cylindrical nanopore with $D_{\mathrm{c}}=d$. Here, the side lengths of the top and bottom pores of the IP-shaped nanopore are respectively defined as $D$ and $d$ (Figure 2). In addition, the optimal conductivity and permittivity of the Si membrane are discussed from the viewpoint of both the throughput and the $\mathrm{S} / \mathrm{N}$ ratio of the ionic current blockade. In this study, the electric field gradients in nanopore devices are examined using a multiphysics model simulation constructed from hydromechanics, electromagnetics, and ionic transport theory [16,17], and we also discuss the availability and a fabrication method for the IP-shaped nanopore from the perspective of high-throughput nanopore devices.

(a)

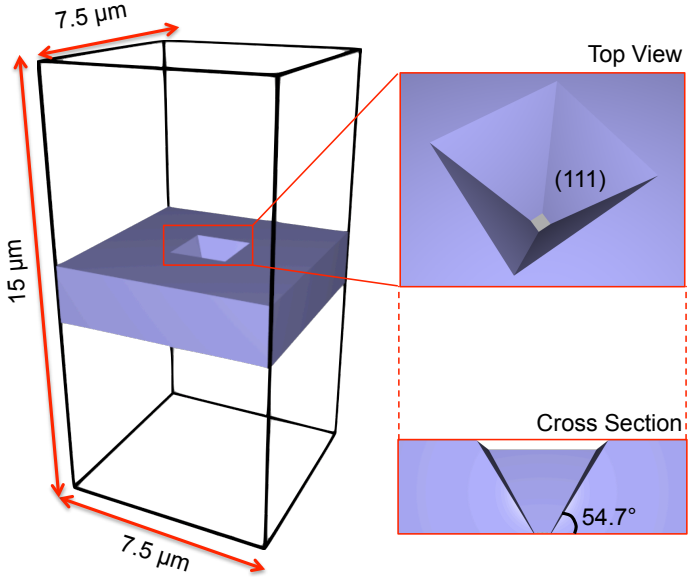

(b)



Figure 2. Simulation model for the inverted pyramid (IP)-shaped nanopore devices. (a) The three-dimensional IP-shaped nanopore model with $D=2932 \mathrm{~nm}$ and $d=100 \mathrm{~nm}$. The $x y$-size and height for the cis and trans chambers are $7.5 \times 7.5 \mu \mathrm{m}$ and $6.5 \mu \mathrm{m}$, respectively. As the IP-shape nanopore is envisioned to be fabricated by an anisotropic wet etching on a (100) silicon wafer, the slope angle is $54.7^{\circ}$. (b) Definitions of the model size, the coordinate origin, the bias voltage, and the concentration of phosphate-buffered saline (PBS) buffer.

\section{Methods}

To begin, we established a multiphysical model to evaluate the electric field gradients. The model consists of three physical equations (Poisson-Boltzmann, Nernst-Plank, and Navier-Stokes) to simulate the electric field at a steady state and its static charges [16,17]. All calculations were performed using COMOL Multiphysics. The three-dimensional models were employed to simulate the electric field. The $x y$-size and height for the cis and trans chambers were $7.5 \times 7.5 \mu \mathrm{m}$ and $6.5 \mu \mathrm{m}$, respectively, and a 2 - $\mu \mathrm{m}$-thick Si membrane (a conductivity of $4.3 \times 10^{-4} \mathrm{~S} / \mathrm{m}$ and a relative permittivity $\left(\varepsilon_{\mathrm{r}}\right)$ of 11.7 were used for nondoped Si [18,19]) with an IP-shaped nanopore of $D=2932 \times 2932 \mathrm{~nm}$ and $d=100 \times 100 \mathrm{~nm}$ was placed between the cis and trans chambers as shown in Figure 2a. In some cases, a $\mathrm{Si}_{3} \mathrm{~N}_{4}$ layer (a conductivity of $1.0 \times 10^{-14} \mathrm{~S} / \mathrm{m}$ and a relative permittivity $\varepsilon_{\mathrm{r}}$ of 6.9 were used for $\left.\mathrm{Si}_{3} \mathrm{~N}_{4}[20,21]\right)$ was placed on the $\mathrm{Si}$ membrane. This pyramidal shape is a well-known structure because it can be fabricated by an anisotropic wet etching on a (100) silicon wafer. This chemical etching provides a slope with the angle of $54.7^{\circ}$ due to the side (111) planes, meaning that the angle is restricted by the etching process and the crystal structure of silicon. Although it is uncertain whether $54.7^{\circ}$ is the optimal angle for the nanopore devices, the angle was employed in this study. In the present calculations, the coordinate's origin was located in the center of each dimension, and the topside corresponds to the ground. The bias voltage of $1.0 \mathrm{~V}$ was applied to the bottom terminal side, and a 137-mmol/L-phosphate-buffered saline (PBS) buffer was also employed as an electrolyte solution (Figure $2 b$ ). Compared with conventional cylindrical nanopores, the electric fields in models consisting of a 100-300-nm-thick $\mathrm{Si}_{3} \mathrm{~N}_{4}$ with a 100-nm-diameter pore structure were also simulated (Figure A1). 


\section{Results and Discussion}

\subsection{Electric Field Gradients in Nanopore Devices}

For the nondoped Si-based IP-shaped nanopore structure, the potential drop is localized inside the structure (Figure 3a), however the electric field exists between the electrode and the entrance of the IP-shaped nanopore (Figure $3 b$ (blue)). This result could be due to the large pore entrance structure, i.e., reducing the $R_{\text {pore, }}$ and relatively smaller resistance and larger permittivity of nondoped Si compared to those of $\mathrm{Si}_{3} \mathrm{~N}_{4}$. Interestingly, the potential drop inside a nanopore shows a nonlinear property, as shown in Figure $3 b$ (blue). Since the pore resistance increases with decreasing the cross-sectional area of the pore (see Equation (1)), the resistance inside the IP-shaped nanopore depends on $z$, namely, the $R_{\text {pore }}$ of the structure is described as:

$$
R_{\text {pore }}=\int_{-\frac{h}{2}}^{\frac{h}{2}} \frac{\alpha}{D(z)^{2}} d z
$$

Thereby, the resistance corresponding to the vicinity of the nanopore exit is considerably higher than that of the vicinity of the nanopore entrance and the surface, resulting in the nonlinear potential drop. From the viewpoint of the equivalent circuit, the pore resistance of the IP-shaped nanopore is described as $\Sigma R^{\mathrm{n}}$ pore $=R_{\text {pore }}^{1}+R^{2}$ pore $+R^{3}$ pore $\ldots R^{\mathrm{P}}$ pore, where $R^{\mathrm{P}}$ pore corresponds to the principal pore resistance causing the potential drop at the bottom of the nanopore (Figure $3 \mathrm{c}$ ). The gradient of the resistance thus causes the potential curve (Figure $3 b$ ).

In contrast, the dispersion of the electric field in the doped Si-based IP-shaped nanopore structure shows relatively large gradients with increasing a conductivity and/or permittivity of the Si membrane, as shown in Figure $3 \mathrm{a}, \mathrm{b}$ (green, red). In these instances, a conductivity of 1.0 and $10^{5} \mathrm{~S} / \mathrm{m}$, and a relative permittivity of 11.7, and 20 were used for the doped $\mathrm{Si}$, respectively. These physical properties are obtained by phosphorus doping with a concentration of $4.4 \times 10^{13}$ and $8.2 \times 10^{19} \mathrm{~cm}^{-3}$, respectively $[19,22]$. Interestingly, the doped Si-based IP-shaped nanopore with $\varepsilon_{\mathrm{r}}=11.7$ shows almost the same potential drop to that of nondoped Si structure. This could be because the permittivity of Si is almost independent from the conductivity in the cases with less than $10^{4} \mathrm{~S} / \mathrm{m}$ [19]. These homogeneous electric field gradients are suitable to drive analytes by electrophoresis. However, the vectors of the force felt by a negative charged analyte, which are opposite directions of the electric field, in a Si-based IP-shaped nanopore device are aligned along the $z$-axis (Figure $4 a, b$ ), meaning that only a small number of analytes pass through a nanopore because many of the translocated analytes along the field are ultimately captured on the membrane surface. To control the vectors, we next employed 50-nm-thick $\mathrm{Si}_{3} \mathrm{~N}_{4}$ layers on the surface of the doped $\mathrm{Si}$ membranes, as shown in Figure 3a. It is interesting to note that the $\mathrm{Si}_{3} \mathrm{~N}_{4}$ layer was found to change the vectors of the force felt by a negatively charged analyte near the $\mathrm{Si}_{3} \mathrm{~N}_{4}$ layer surface in the direction of the IP-shaped nanopore, and the potential drops in the entire cis chamber remained, as shown in Figure 3b (purple and orange) and Figure 4c,d. These results strongly suggest that most of the analytes suspended in the cis chamber can pass through a nanopore by electrophoresis, even though analytes are suspended far away from the nanopore entrance. Indeed, the electric field exists $7500 \mathrm{~nm}$ above the nanopore center for each device, suggesting that the capture radius is more than $7500 \mathrm{~nm}$ in these device structures, which is 50 times larger than that of the conventional nanopore with $d=100 \mathrm{~nm}$ and $h=100 \mathrm{~nm}$ (Figure A1). Therefore, in a simple estimation, only taking into account the effect due to the electric field, a $12.5 \times 10^{4}\left(=50^{3}\right)$ times lower analyte concentration is the available concentration for the IP-shaped nanopore compared with the conventional nanopore. This improvement in the throughput is also comparable to that of array structures of $10^{4}$ nanopores per $\mathrm{mm}^{2}$ [11]. However, this possibility deserves careful examination because the electroosmotic flow in a device also affects the throughput [23]. Figure 5 shows simulated results of electroosmotic flow velocity distributions in $\mathrm{Si}_{3} \mathrm{~N}_{4} /$ doped Si-based $\left(\varepsilon_{\mathrm{r}}=11.7\right)$ IP-shaped nanopore (Figure $5 \mathrm{a}-\mathrm{c}$ ) and a conventional $\mathrm{Si}_{3} \mathrm{~N}_{4}$ cylindrical nanopore (Figure $5 \mathrm{~d}$ ) device. For the 
IP-shaped nanopore, the electroosmotic flow does not seem to obstruct the analyte translocations from the cis to the trans chamber because the main flow is in the same direction of the vectors of the force felt by a negative charged analyte and in the perpendicular direction to the membrane (Figure 5a), whereas the $\mathrm{Si}_{3} \mathrm{~N}_{4}$ cylindrical nanopore causes the electroosmotic flow in the opposite direction of the analyte translocations because of the negative surface charge of the $\mathrm{Si}_{3} \mathrm{~N}_{4}$, which is a well-known behavior (Figure 5d) [24]. In the case of the IP-shaped nanopore, the electroosmotic flow in the opposite direction to that in the conventional nanopore seems to be caused by the vortical flows in the vicinity of pore entrance edges (Figure $5 b$ ). Since the $\mathrm{Si}_{3} \mathrm{~N}_{4}$ layer on the IP-shaped Si membrane causes the electroosmotic flow in the opposite direction to analyte translocations near the $\mathrm{Si}$ and $\mathrm{Si}_{3} \mathrm{~N}_{4}$ surfaces (Figure 5c), these electroosmotic flows could finally result in the vortical flows, as shown in Figure $5 \mathrm{~b}$. However, it is to be noted that the dynamics of the electroosmotic flow in the IP-shaped nanopore should be examined for each device because the dynamics in a conical pore structure are found to depend on the salt concentration in a buffer solution, the Debye length on the sidewall inside a nanopore, and the structure size [23,25]. It is reported that the vortical flow is generated inside a nanopore, which affects the device throughput, especially in a small pyramidal nanopore structure [23]. For the present IP-shaped nanopore device consisting of 137-mmol/L-PBS buffer, as the velocity of the electroosmotic flow is ca. $1.0 \times 10^{-5} \mathrm{~m} / \mathrm{s}$ in the vicinity of the $\mathrm{Si}_{3} \mathrm{~N}_{4}$ surface, the analytes around the $\mathrm{Si}_{3} \mathrm{~N}_{4}$ layer surface with $q E t / m<1.0 \times 10^{-5} \mathrm{~m} / \mathrm{s}$ cannot approach the nanopore, where $q, E, t$, and $m$ denote the charge of the analytes, the electric field, time, and the mass of the analytes, respectively. Therefore, although the device throughput depends not only on the electric field and the electroosmotic flow but also the analyte physical properties, these simulation results indicate that the $\mathrm{Si}_{3} \mathrm{~N}_{4} / \mathrm{Si}$-based IP-shaped nanopore is one of the suitable device structures for high-throughput sensing.

We next discuss the $\mathrm{Si}_{3} \mathrm{~N}_{4} / \mathrm{Si}$-based IP-shaped nanopores from the viewpoint of the ionic current blockade. Here, current blockades caused by a translocation of an insulating nanoparticle with 30-nm-diameters were simulated for the IP-shaped nanopores consisting of the Si membrane with $\varepsilon_{\mathrm{r}}=11.7$ and 20. As shown in Figure $6 \mathrm{a}, \mathrm{b}$, the intensities of the current blockade $\left(I_{\mathrm{p}}\right)$ were found to be 4 $\mathrm{nA}$ and $6 \mathrm{nA}$ for the nanopore structures with $\varepsilon_{\mathrm{r}}=11.7$ and 20, respectively, although the ratio of $I_{\mathrm{p}}$ to the base line current $\left(I_{\mathrm{b}}\right)$ is almost the same for both structures. However, the widths of resistive pulses show a dependence on $\varepsilon_{\mathrm{r}}$, as show in Figure $6 \mathrm{a}, \mathrm{b}$. In the case of the nanopore with $\varepsilon_{\mathrm{r}}=11.7$, the ionic current is decreased by the nanoparticle suspended farther away from the bottom of the IP-shaped structure compared to the case of the nanopore with $\varepsilon_{\mathrm{r}}=20$. Since the ionic current blockade is caused by a nanoparticle excluding ions inside a nanopore during translocation, the difference in the widths of the current signal could arise from that the ion density in the nanopore structure with $\varepsilon_{\mathrm{r}}=11.7$ is distributed more broadly than that in the structure with $\varepsilon_{\mathrm{r}}=20$ (Figure $6 \mathrm{c}$ ). These results indicate that the IP-shaped nanopore structures consisting of a $\mathrm{Si}_{3} \mathrm{~N}_{4} / \mathrm{Si}$ layer can be utilized for nanopore devices as well as the conventional nanopore structures. As the structure with higher-doped Si membrane shows higher $I_{p}$, a high-doped Si membrane is potentially suitable material for the IP-shaped nanopore structure when only considering the peak intensity $I_{\mathrm{p}}$.

As a qualitative explanation, the combination of the insulating layer on the semiconducting membrane and the IP-shaped nanopore structure with the large entrance plays a key role in the formation of the suitable electric field gradient within a nanopore device in terms of the high-throughput. Indeed, the electric field is localized inside a nanopore for cylindrical Si nanopore with a $\mathrm{Si}_{3} \mathrm{~N}_{4}$ layer (Figure S1). This result would be understood using an equivalent circuit. The simplified resistance corresponding to the IP-shaped nanopore structure is equivalent to two resistances in a series circuit, i.e., $\Sigma R^{\mathrm{n}}$ pore and $R^{\mathrm{P}}$ pore, and the $\mathrm{Si}_{3} \mathrm{~N}_{4} / \mathrm{Si}$ layers correspond to a series of connected $R C$ parallel circuits (Figure $3 \mathrm{~d}$ ). It is notable that high resistances due to $\mathrm{Si}_{3} \mathrm{~N}_{4}\left(R_{\mathrm{N}}\right)$ and $R^{\mathrm{P}}$ pore, which are placed in parallel in the other equivalent circuits and are the main factors causing the drastic potential drop, are separated from each other, as shown Figure 3d. This fact means that the drastic potential drop is separated into two steps corresponding to $R_{\mathrm{N}}$ and $R^{\mathrm{P}}$ pore, resulting in the formation of the suitable electric field gradient within a nanopore device. 
(a)

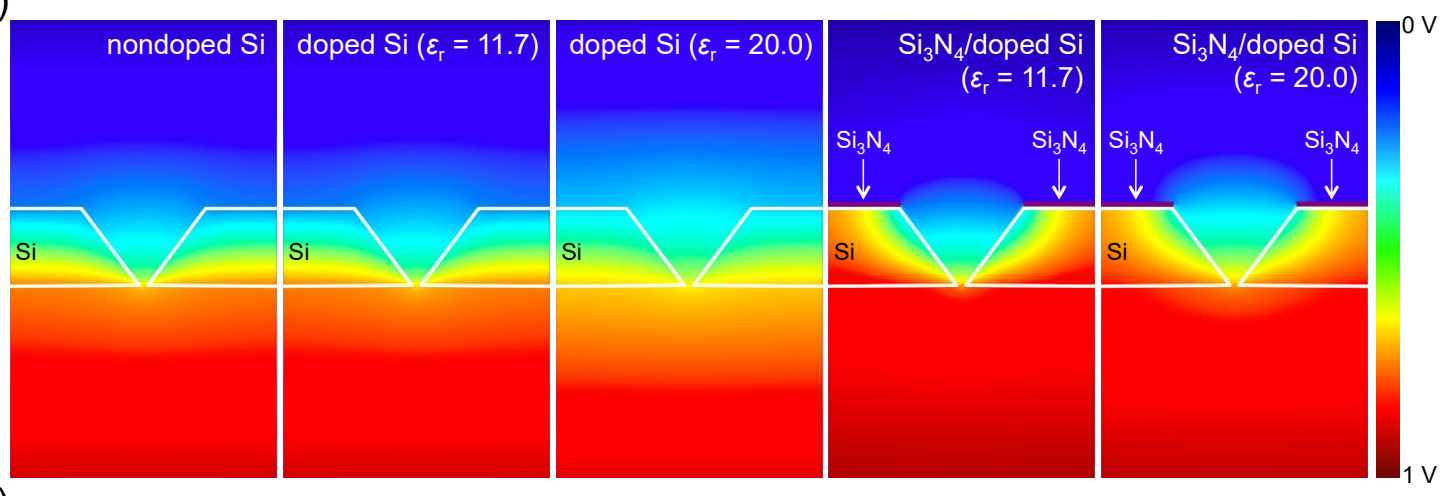

(b)

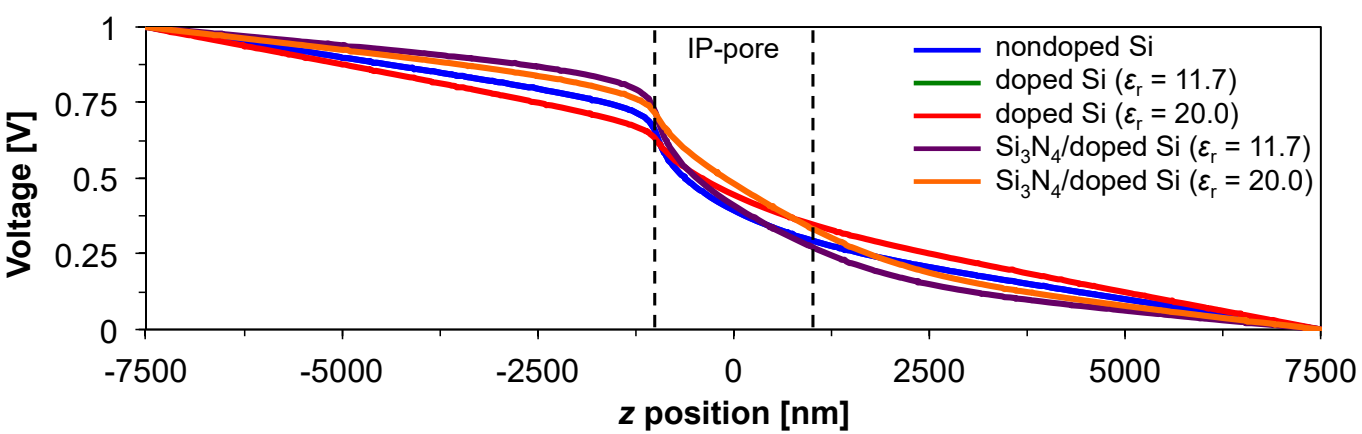

(c)

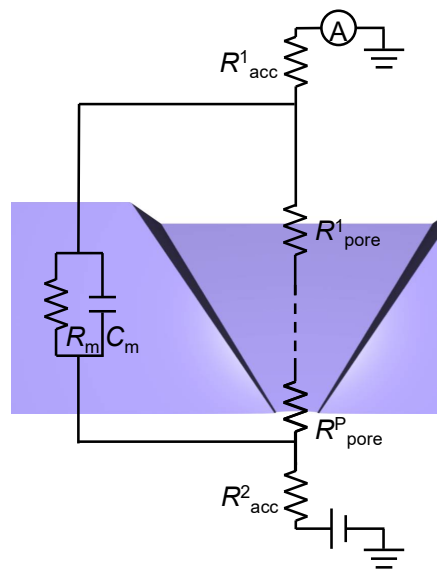

(d)

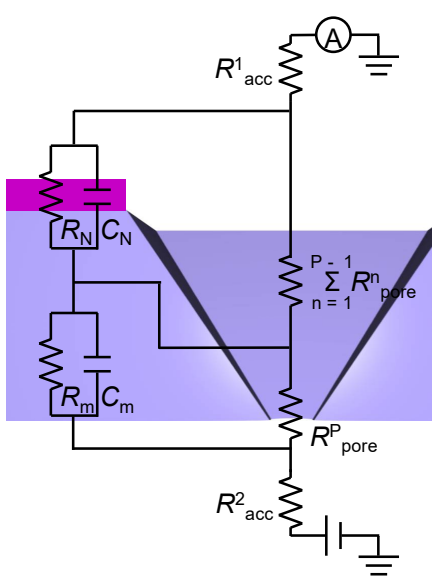

Figure 3. Simulation results for the IP-shaped nanopore structures. (a) Cross-sectional potential distributions for the nondoped $\mathrm{Si}$, the doped $\mathrm{Si}$, and the $\mathrm{Si}_{3} \mathrm{~N}_{4}$ /doped $\mathrm{Si}$ nanopores. The potential drop was found to depend on the structure and the material permittivity. (b) Potential dependencies on the $z$ position on the center axis $(x=0, y=0)$ for the nondoped $\mathrm{Si}$ (blue), the doped $\mathrm{Si}\left(\varepsilon_{\mathrm{r}}=11.7\right)$ (green), the doped $\mathrm{Si}\left(\varepsilon_{\mathrm{r}}=20\right)(\mathrm{red})$, the $\mathrm{Si}_{3} \mathrm{~N}_{4} /$ doped $\mathrm{Si}\left(\varepsilon_{\mathrm{r}}=11.7\right)\left(\right.$ purple), and the $\mathrm{Si}_{3} \mathrm{~N}_{4} /$ doped $\mathrm{Si}\left(\varepsilon_{\mathrm{r}}=20\right)$ (orange) nanopores. Here, as the dependences of the nondoped Si and the doped Si $\left(\varepsilon_{\mathrm{r}}=11.7\right)$ are almost the same property, the potential curves are overlapped. (c) Equivalent circuit of the Si-based IP-shaped nanopore. (d) Equivalent circuit of the $\mathrm{Si}_{3} \mathrm{~N}_{4} / \mathrm{Si}$-based IP-shaped nanopore.

The $\mathrm{Si}_{3} \mathrm{~N}_{4} / \mathrm{Si}$-based IP-shaped nanopore structure is thus expected to improve the throughput of the nanopore devices. However, we should discuss the $\mathrm{S} / \mathrm{N}$ ratio of the IP-shaped nanopore devices, because the large capacitance of the $\mathrm{Si}\left(C_{\mathrm{m}}\right)$ is predicted to cause greater noise in the ionic current. In the case of the $\mathrm{Si}_{3} \mathrm{~N}_{4} / \mathrm{Si}$-based IP-shaped nanopore, the total capacitance is estimated to be $C_{\text {total }}=\left(C_{\mathrm{N}}\right.$ $\left.\times C_{\mathrm{m}}\right) /\left(C_{\mathrm{N}}+C_{\mathrm{m}}\right)$, where $C_{\mathrm{N}}$ denotes the capacitance of the $\mathrm{Si}_{3} \mathrm{~N}_{4}$ layer, because each capacitance corresponding to the $\mathrm{Si}_{3} \mathrm{~N}_{4}$ and the $\mathrm{Si}$ is in a series circuit, meaning a smaller capacitance of the $\mathrm{Si}_{3} \mathrm{~N}_{4} / \mathrm{Si}$ layers than that of a individual single-doped $\mathrm{Si}$ membrane and $\mathrm{Si}_{3} \mathrm{~N}_{4}$ layer (Figure 3d). The noise 
level comparison of the $\mathrm{Si}_{3} \mathrm{~N}_{4} / \mathrm{Si}$-based IP-shaped nanopore and the conventional $\mathrm{Si}_{3} \mathrm{~N}_{4}$ nanopore is experimentally examined in the next section.

(a)

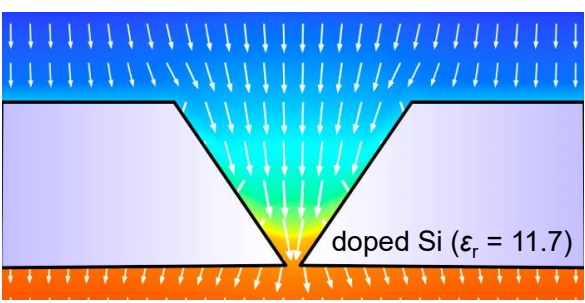

(c)

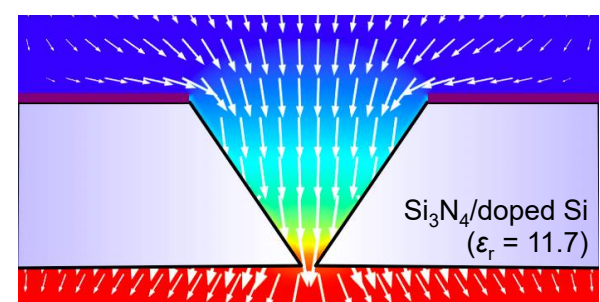

(b)



(d)

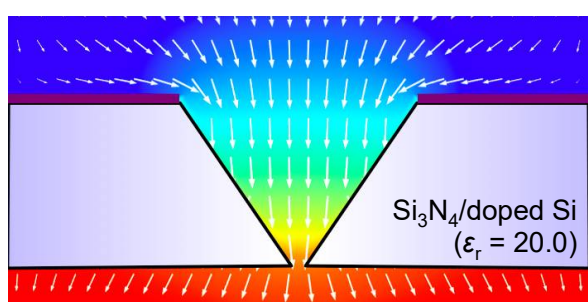

Figure 4. Simulation results of the vectors of the force felt by a negative charged analyte, which are the opposite directions of the electric field, in nanopore devices. (a) Doped $\mathrm{Si}\left(\varepsilon_{\mathrm{r}}=11.7\right)$. (b) Doped $\mathrm{Si}\left(\varepsilon_{\mathrm{r}}=20\right)$. (c) $\mathrm{Si}_{3} \mathrm{~N}_{4} /$ doped $\mathrm{Si}\left(\varepsilon_{\mathrm{r}}=11.7\right)$. (d) $\mathrm{Si}_{3} \mathrm{~N}_{4}$ /doped $\mathrm{Si}\left(\varepsilon_{\mathrm{r}}=20\right)$. $\mathrm{Si}_{3} \mathrm{~N}_{4}$ layer changes the orientation of the vectors near the $\mathrm{Si}_{3} \mathrm{~N}_{4}$ layer surface in the direction of the IP-shaped nanopore.

(a)

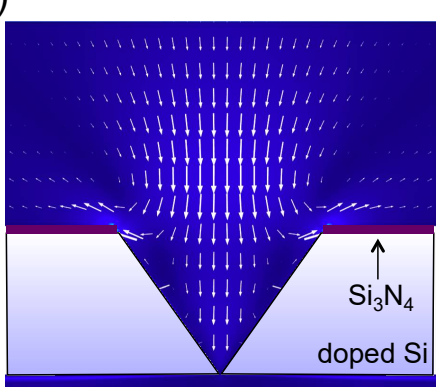

(c)



(b)

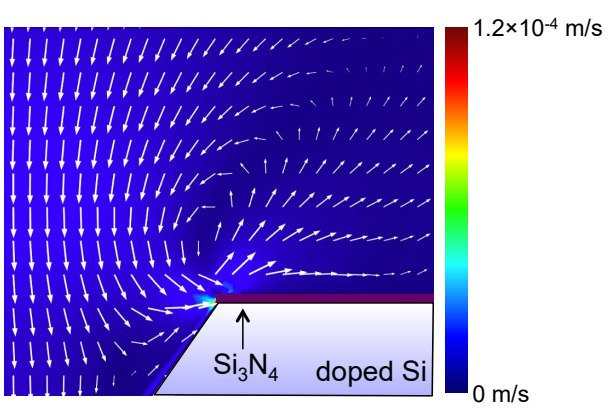

(d)

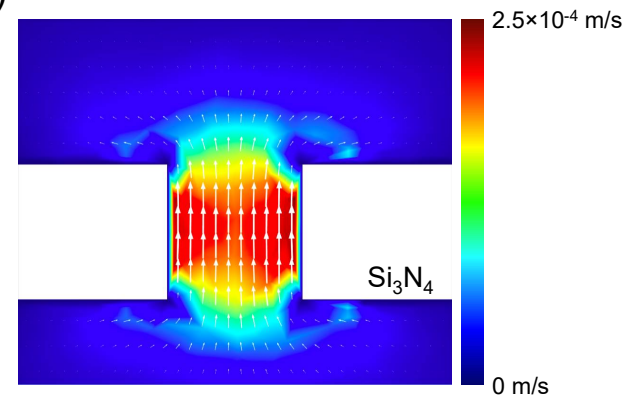

Figure 5. Simulated electroosmotic flows. (a) Electroosmotic flow velocity distribution in the $\mathrm{Si}_{3} \mathrm{~N}_{4}$ /doped $\mathrm{Si}\left(\varepsilon_{\mathrm{r}}=11.7\right)$ IP-shaped nanopore device. $(\mathbf{b}, \mathbf{c})$ Magnified views of the distribution in the $\mathrm{Si}_{3} \mathrm{~N}_{4}$ /doped Si IP-shaped nanopore device. (d) Electroosmotic flow velocity distribution in the cylindrical $\mathrm{Si}_{3} \mathrm{~N}_{4}$ nanopore device. The electroosmotic flow in the IP-shaped nanopore seems to cause positive effects for the device throughput. 
(a)

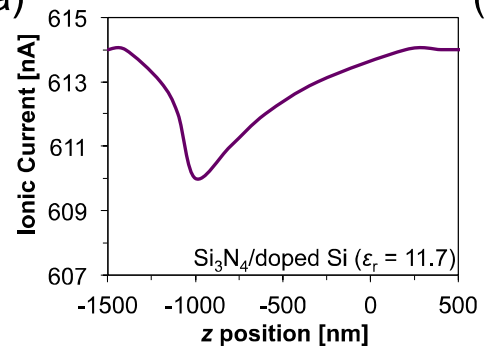

(b)



(c)

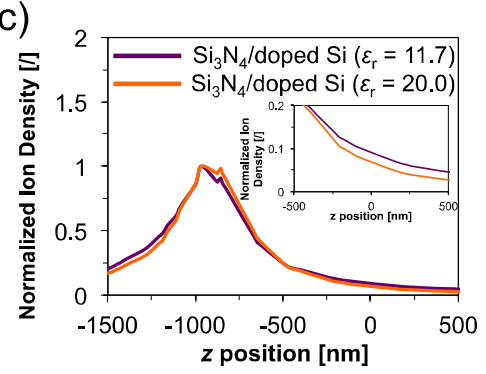

Figure 6. Simulated ionic current blockades. (a) Ionic current dependence in $z$ position of a 30-nm-diameter nanoparticle in the $\mathrm{Si}_{3} \mathrm{~N}_{4}$ /doped $\mathrm{Si}\left(\varepsilon_{\mathrm{r}}=11.7\right)$ nanopore. (b) Ionic current dependence in $z$ position of a 30-nm-diameter nanoparticle in the $\mathrm{Si}_{3} \mathrm{~N}_{4}$ /doped $\mathrm{Si}\left(\varepsilon_{\mathrm{r}}=20\right)$ nanopore. (c) Normalized dependences of ion density in $z$ position in each nanopore device and the extended figure of the dependences in $z=-500-500 \mathrm{~nm}$ (inset).

\subsection{Fabrications and Ionic Current Noise Level of the IP-Shaped Nanopore Devices}

Finally, we discuss fabrications and the ionic current noise level of the IP-shaped nanopore that facilitate high-throughput nanopore devices. As mentioned above, the IP-shape with $\theta=54.7^{\circ}$ can be fabricated by an anisotropic wet etching on a (100) silicon wafer. However, controlling the nanopore size $(d)$ is difficult using this method because the thickness of $S i$ wafer usually has a margin of error of about \pm ca. $10 \%$. Si wafers with a nanometer thickness are thus preferred for the nanopore fabrications with an accuracy of a couple of nanometers. However, the higher thickness of the Si membrane $(h)$ is a more appropriate structure to improve the device throughput, because a higher thickness causes a more gradual potential drop in a nanopore device (Figure A2). This fact means that the difficulty of the fabrication increases with $h$ due to the increasing error thickness with $h$. Hence, the Si-based IP-shaped nanopore structures with micrometer thicknesses have not been reported, though structures with nanometer thicknesses are well-known $[26,27]$. Indeed, S. Zeng et al. recently report pyramidal nanopore structures with a ca. $80 \mathrm{~nm}$ thickness [23]. To fabricate the IP-shaped nanopore structures with micrometer thicknesses, we employed a two-step etching process in this study (Figure 7a).

In this study, 300- $\mu$ m-thick $S i$ wafers, both sides of which were covered with 50 -nm-thick $\mathrm{Si}_{3} \mathrm{~N}_{4}$ membranes, were employed as a substrate. First, 390- $\mu \mathrm{m}$-square and 1.0-mm-square windows on the top and the bottom sides of the $\mathrm{Si}_{3} \mathrm{~N}_{4}$ membrane were respectively fabricated by photolithography (Figure 7a-2). The topside $\mathrm{Si}_{3} \mathrm{~N}_{4}$ window was wet-etched in a $\mathrm{KOH}$ solution at $120^{\circ} \mathrm{C}$ for $60 \mathrm{~min}$ (step I), resulting in a 275.4- $\mu \mathrm{m}$-height IP-shape (Figure 7a-3). After that, a 20-nm-thick $\mathrm{Cr}$ film was deposited as a protection film by sputtering on the etched area (Figure 7a-4), and subsequently the bottom side window was wet-etched in a $\mathrm{KOH}$ solution at $30{ }^{\circ} \mathrm{C}$ with a slow etching rate (step II), and the etching went so far as to reach the $\mathrm{Cr}$ film (Figure 7a-5). Here, reaching the $\mathrm{Cr}$ film was confirmed by an alternating scanning electron microscope (SEM) observation and the slow $\mathrm{KOH}$ etching. Finally, the $\mathrm{Si}_{3} \mathrm{~N}_{4} / \mathrm{Si}$-based IP-shaped nanopore structure with $D=390 \mu \mathrm{m}$ and $h=275 \mu \mathrm{m}$ was obtained by removing the $\mathrm{Cr}$ film, as shown in Figure $\mathrm{7b}$. Because the two-step etching process enabled us to fabricate the nanopore structures without electron beam lithography, the present fabrication method is preferable from the perspective of fabrication costs. However, the fabrication of a square-shaped nanopore with $d=100 \mathrm{~nm}$ was found to be difficult using this method because the etching rate is not exactly the same among each (111) surface of silicon. These experimental errors of the etching rate result in a rectangular-shaped nanopore with a width of $100 \mathrm{~nm}$. Thus, a nanopore with $d=500 \mathrm{~nm}$ is currently the minimum square-shaped structure using this method. However, since the electric field properties of the IP-shaped nanopore are almost independent from the pore size, the 500-nm IP-shaped nanopore would also demonstrate high-throughput sensing (Figure S2). 
(a)

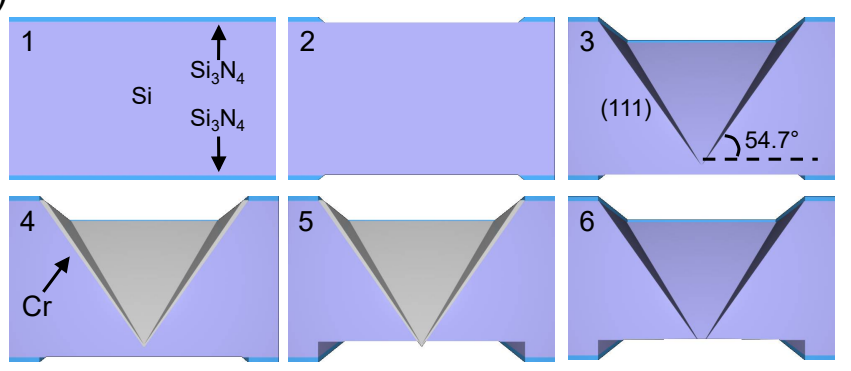

(b)

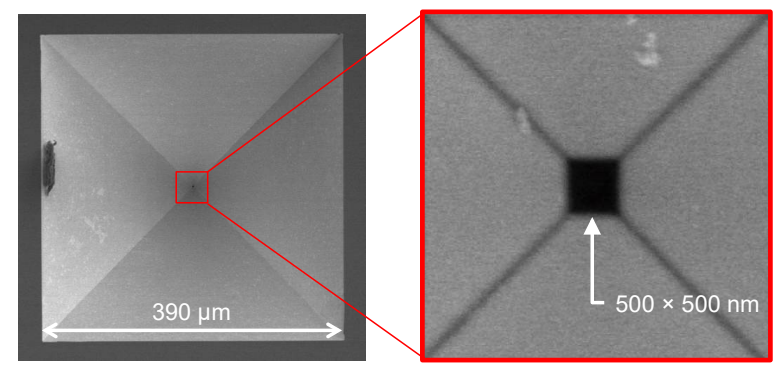

Figure 7. Fabrication process for the $\mathrm{Si}_{3} \mathrm{~N}_{4} / \mathrm{Si}$-based IP-shaped nanopore structures and scanning electron microscope (SEM) images of the nanostructures. (a) Schematic illustrations of the fabrication process. The two-step etching processes (No. 3 and 5) enabled us to fabricate the nanostructures without electron beam lithography. (b) SEM images of the $\mathrm{Si}_{3} \mathrm{~N}_{4} / \mathrm{Si}$-based IP-shaped nanopore structures with $D / d=$ ca. 1000 fabricated using the present method.

Figure 8 shows the noise level comparison of the $\mathrm{Si}_{3} \mathrm{~N}_{4} / \mathrm{Si}$-based IP-shaped nanopore and the conventional cylindrical $\mathrm{Si}_{3} \mathrm{~N}_{4}$ nanopore. Here, a phosphorus-doped $\mathrm{Si}$ wafer with $5 \mathrm{~S} / \mathrm{m}$ and 137-mmol/L-PBS buffer were employed as a Si membrane and buffer solution, respectively, and an electrophoretic field of $100 \mathrm{mV}$ was applied to the pore, utilizing two $\mathrm{Ag} / \mathrm{AgCl}$ electrodes. The ionic current was detected at $1 \mathrm{MHz}$ by employing a home-built current amplifier backed by a digitizer (NI-5922, National Instruments, Austin, TX, USA) and stored in a RAID hard drive (HDD-8265, National Instruments, Austin, TX, USA) under LabVIEW control [7]. Thereafter, $1 \mathrm{MHz}$ ionic current-time data were finally compressed to $100 \mathrm{kHz}$ and normalized at $0 \mathrm{~A}$ to compare the peak-to-peak noise level between the two ionic current data. As shown in Figure 8, the peak-to-peak level of ionic current for the IP-shaped nanopores is lower than that of conventional nanopores. This result indicates that the total capacitance of the IP-shaped nanopore structure $C_{\text {total }}$ is smaller than that of conventional $\mathrm{Si}_{3} \mathrm{~N}_{4}$ nanopore structure $C_{\mathrm{N}}$. Indeed, $C_{\text {total }}=\left(C_{\mathrm{N}} \times C_{\mathrm{m}}\right) /\left(C_{\mathrm{N}}+C_{\mathrm{m}}\right)$ and $C_{\mathrm{N}}$ are estimated to be $C_{\text {total }}=2.16 \times$ $10^{-10} \mathrm{~F}$ and $C_{\mathrm{N}}=7.64 \times 10^{-7} \mathrm{~F}$, respectively, as $\mu_{\mathrm{N}}=6.109 \times 10^{-11} \mathrm{~F} / \mathrm{m}, S_{\mathrm{N}}=6.25 \times 10^{-4} \mathrm{~m}^{2}, h_{\mathrm{N}}=5.0 \times$ $10^{-8} \mathrm{~m}, C_{\mathrm{m}}=2.158 \times 10^{-10} \mathrm{~F}, \mu_{\mathrm{m}}=1.036 \times 10^{-10} \mathrm{~F} / \mathrm{m}, S_{\mathrm{m}}=6.25 \times 10^{-4} \mathrm{~m}^{2}, h_{\mathrm{m}}=3.00 \times 10^{-4} \mathrm{~m}$. Here, $\mu_{\mathrm{N}}, \mu_{\mathrm{m}}, S_{\mathrm{N}}, S_{\mathrm{m}}, h_{\mathrm{N}}$, and $h_{\mathrm{m}}$ correspond to the permittivity, the area, and the thickness of $\mathrm{Si}_{3} \mathrm{~N}_{4}$ layer $(\mathrm{N})$ and Si membrane $(\mathrm{m})$, respectively. 
(a)

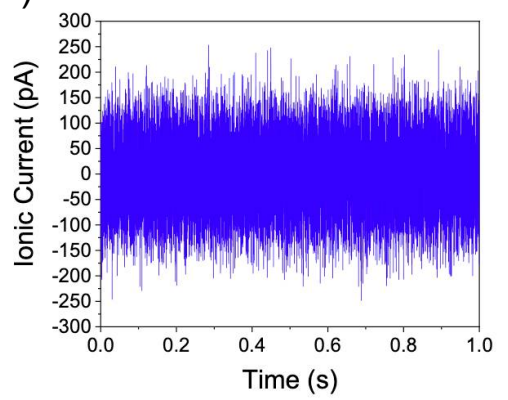

(b)



Figure 8. Noise level of the ionic currents. (a) Ionic current in a conventional $\mathrm{Si}_{3} \mathrm{~N}_{4}$ nanopore. (b) Ionic current in a $\mathrm{Si}_{3} \mathrm{~N}_{4}$ /doped Si-based IP-shaped nanopore, i.e., doped Si-based IP-shaped nanopore with a $\mathrm{Si}_{3} \mathrm{~N}_{4}$ layer. Here, the central value of each current is normalized at $0 \mathrm{~A}$. It was found that peak-to-peak noise level of the IP-shaped nanopore is lower than that of the conventional cylindrical $\mathrm{Si}_{3} \mathrm{~N}_{4}$ nanopore.

\section{Conclusions}

$\mathrm{Si}_{3} \mathrm{~N}_{4} / \mathrm{Si}$-based IP-shaped nanopore structures, which could be fabricated only by photolithography, were proposed as structures for high-throughput nanopore devices. The insulating $\mathrm{Si}_{3} \mathrm{~N}_{4}$ layer on the doped Si membrane and the large entrance of the IP-shaped nanopore structure were found to cause a three-dimensionally homogeneous potential drop in the entire cis chamber, and the electric field distributed in the direction of the nanopore entrance in the vicinity of the $\mathrm{Si}_{3} \mathrm{~N}_{4}$ layer surface. These modifications of the electric field increase the capture radius for analytes, suggesting higher throughput for the presented devices. The homogeneous potential drop within the IP-shaped nanopore could provide another advantage from the perspective of the sensing accuracy of the nanopore devices because the homogeneous potential drop causes slower translocation speed of analytes compared with the drastic potential drop in the conventional cylindrical nanopores. In some cases of nanopore devices, since the translocation speed is too fast to analyze single molecules and/or single bioparticles passing through a nanopore, reducing the analyte translocation speed is the major challenge $[2,7,28]$. Thus, reducing the translocation speed due to the homogeneous potential drop inside the IP-shaped nanopore is expected to improve the sensing accuracy, such as spatial resolution in the shape analysis [7]. However, it is to be noted that the sensing accuracy also depends on the ratio of signal to noise of the ionic current and the shape of nanopore. In addition, the nanopore sensing is currently limited to relatively large molecules, such as long DNA and large proteins, and single bioparticles, such as viruses. Further improvement of the sensing accuracy for the identification of small, single molecules is a subject for future study. The present results suggest a high potential for the $\mathrm{Si}_{3} \mathrm{~N}_{4} / \mathrm{Si}$-based IP-shaped nanopore to serve as a novel device structure that facilitates high-throughput sensing for single molecules and bioparticles; additionally, the present findings present new methods for controlling the electric field in nanofluidics.

Supplementary Materials: The following are available online at http://www.mdpi.com/2072-666X/11/10/893/s1, Figure S1: Electric field in the $\mathrm{Si}_{3} \mathrm{~N}_{4} / \mathrm{Si}$ cylindrical nanopore, Figure S2: Electrical field in the $\mathrm{Si}_{3} \mathrm{~N}_{4} / \mathrm{Si}_{\mathrm{IP}}$-shaped nanopore with $d=500 \mathrm{~nm}$.

Author Contributions: Conceptualization, S.R. and M.T.; methodology, S.R. and R.M.; validation, S.R., R.M., and M.T.; investigation (simulation), S.R.; investigation (experiment), M.T.; writing-original draft preparation, S.R.; writing-review and editing, R.M., and M.T.; visualization, S.R.; supervision, M.T.; funding acquisition, S.R. All authors have read and agreed to the published version of the manuscript.

Funding: This research was funded by the Japan Society for the Promotion of Science (JSPS) KAKENHI, grant number 19K05234, and Japan Science \& Technology Agency (JST) PRESTO, grant number JPMJPR17HC.

Acknowledgments: This research is partially supported by the Japan Society for the Promotion of Science (JSPS) and Japan Science \& Technology Agency (JST) PRESTO. This work is also performed under the Cooperative Research Program of "Dynamic Alliance for Open Innovation Bridging Human, Environment and Materials".

Conflicts of Interest: The authors declare no conflict of interest. 


\section{Appendix A}

(a)

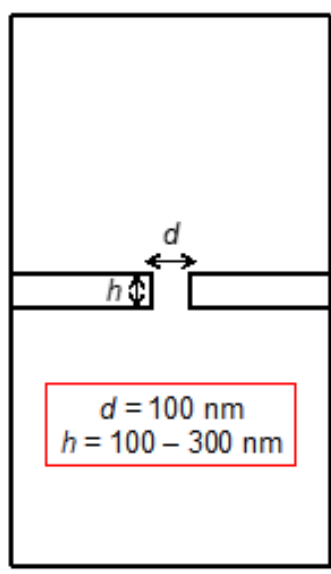

(b)

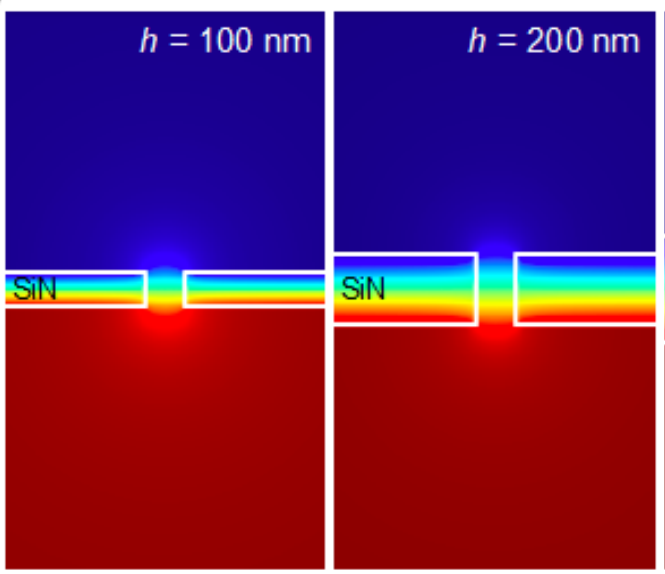

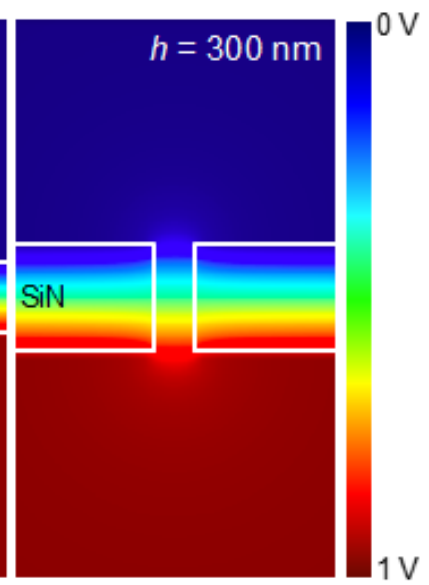

(c)

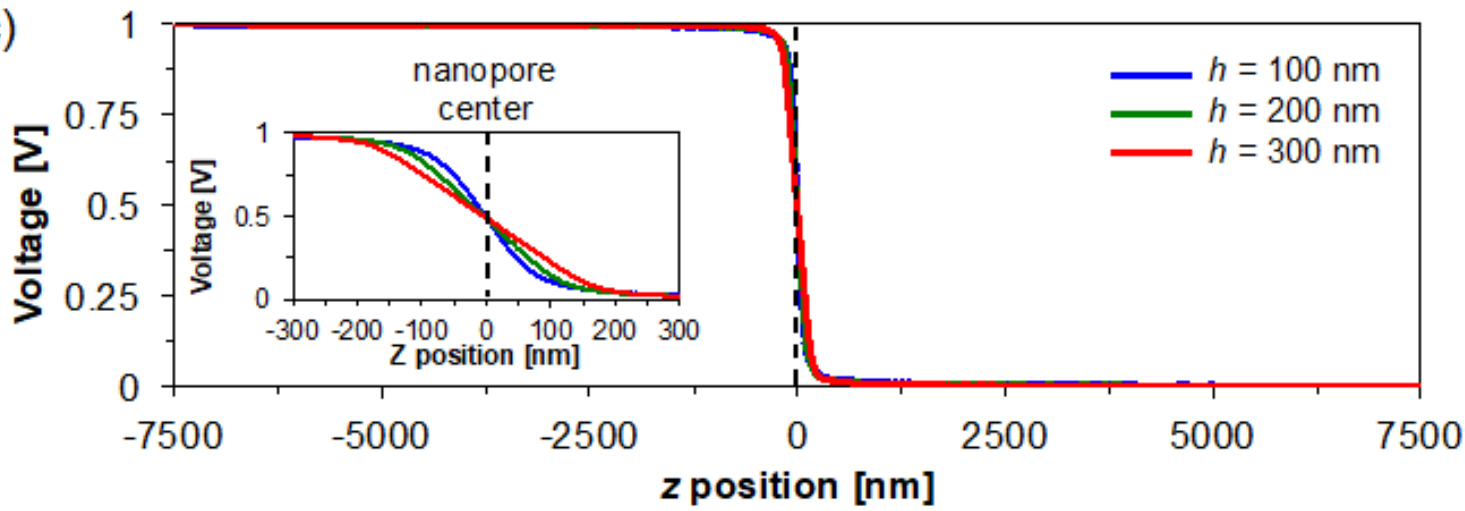

Figure A1. Simulation results for the conventional cylindrical nanopore structures. (a) Schematic illustration of the simulation model. (b) Cross-sectional potential distributions at $y=0$ for the nanopores with $h=100 \mathrm{~nm}, 200 \mathrm{~nm}$, and $300 \mathrm{~nm}$. (c) Potential dependences on the $z$ position on the center axis $(x=0, y=0)$ for the nanopores with $h=100 \mathrm{~nm}$ (blue), $200 \mathrm{~nm}$ (green), and $300 \mathrm{~nm}$ (red), and enlarged view near $z=0$ (inset). The potential drops are respectively $0.55 \mathrm{~V}, 0.69 \mathrm{~V}$, and $0.78 \mathrm{~V}$ inside the nanopores with $h=100 \mathrm{~nm}, 200 \mathrm{~nm}$, and $300 \mathrm{~nm}$. The full electric field gradients of $0-1.0 \mathrm{~V}$ exist within $z=-200-200 \mathrm{~nm}$ in the present structures (Figure A1c), meaning that analytes suspended more than $200 \mathrm{~nm}$ above the nanopore center $(z=0)$ cannot pass through a nanopore by electrophoresis unless analytes at a distance greater than the capture radius diffuse into the region incidentally by Brownian motion. These results are comparable to previous reports about the conventional cylindrical nanopore structures [29]. 


\section{Appendix B}

(a)

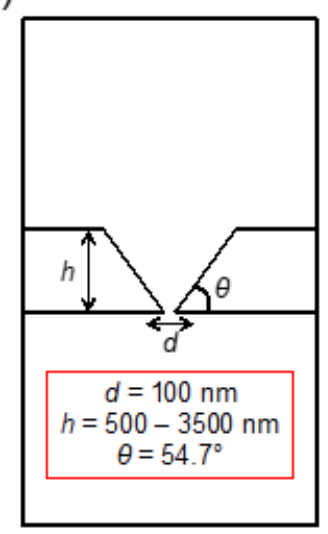

(b)

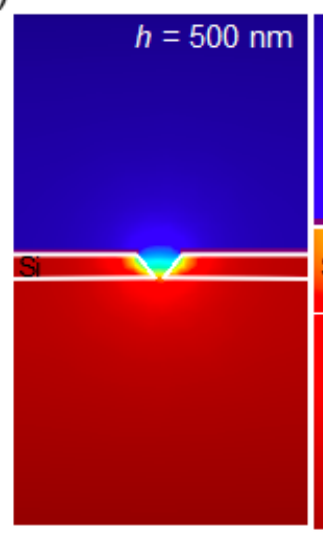

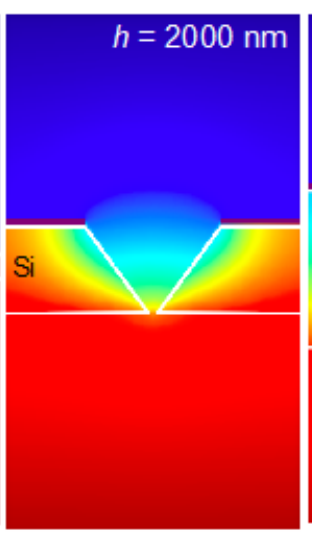



(c)

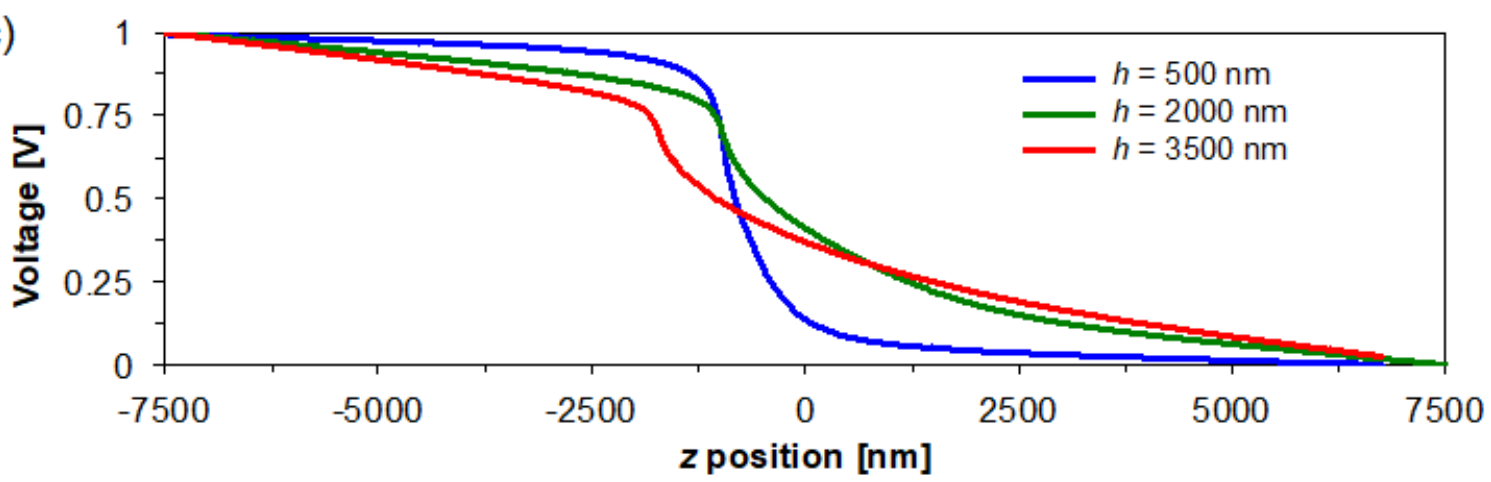

Figure A2. Dependence of the electric field distribution on the thickness of Si membrane for the $\mathrm{Si}_{3} \mathrm{~N}_{4} / \mathrm{Si}$-based nanopore structures. (a) Schematic illustration of the simulation model. (b) Cross-sectional potential distributions for the nanopores with $h=500 \mathrm{~nm}, 2000 \mathrm{~nm}$, and $3500 \mathrm{~nm}$. It was found that higher thickness causes more gradual potential drop in a nanopore device. (c) Potential dependences on $z$ position on the center axis $(x=0, y=0)$ for the nanopores with $h=500 \mathrm{~nm}$ (blue), $2000 \mathrm{~nm}$ (green), and $3500 \mathrm{~nm}$ (red).

\section{References}

1. Cheng, M.H.; Bahar, I. Monoamine Transporters: Structure, Intrinsic Dynamics and Allosteric Regulation. Nat. Struct. Mol. Biol. 2019, 26, 545-556. [CrossRef]

2. Venkatesan, B.M.; Bashir, R. Nanopore Sensors for Nucleic Acid Analysis. Nat. Nanotechnol. 2011, 6, 615-624. [CrossRef]

3. Dekker, C. Solid-State Nanopores. Nat. Nanotechnol. 2007, 2, 209-215. [CrossRef]

4. Tu, B.; Bai, S.; Lu, B.; Fang, Q. Conic shapes have higher sensitivity than cylindrical ones in nanopore DNA sequencing. Sci. Rep. 2018, 8, 9097. [CrossRef]

5. Di Ventra, M.; Taniguchi, M. Decoding DNA, RNA and Peptides with Quantum Tunnelling. Nat. Nanotechnol. 2016, 11, 117-126. [CrossRef]

6. Arima, A.; Tsutsui, M.; Harlisa, I.H.; Yoshida, T.; Tanaka, M.; Yokota, K.; Tonomura, W.; Taniguchi, M.; Okochi, M.; Washio, T.; et al. Selective Detections of Single-Viruses Using Solid-State Nanopores. Sci. Rep. 2018, 8, 16305. [CrossRef]

7. Ryuzaki, S.; Tsutsui, M.; He, Y.; Yokota, K.; Arima, A.; Morikawa, T.; Taniguchi, M.; Kawai, T. Rapid Structural Analysis of Nanomaterials in Aqueous Solutions. Nanotechnology 2017, 28, 155501. [CrossRef]

8. Tsutsui, M.; Hongo, S.; He, Y.; Taniguchi, M.; Gemma, N.; Kawai, T. Single-Nanoparticle Detection Using a Low-Aspect-Ratio Pore. ACS Nano 2012, 6, 3499-3505. [CrossRef] 
9. Bell, N.A.W.; Muthukumar, M.; Keyser, U.F. Translocation Frequency of Double-Stranded DNA through a Solid-State Nanopore. Phys. Rev. E 2016, 93, 1-10. [CrossRef]

10. He, Y.; Gillespie, D.; Boda, D.; Vlassiouk, I.; Eisenberg, R.S.; Siwy, Z.S. Tuning Transport Properties of Nanofluidic Devices with Local Charge Inversion. J. Am. Chem. Soc. 2009, 131, 5194-5202. [CrossRef]

11. Huang, S.; Romero-Ruiz, M.; Castell, O.K.; Bayley, H.; Wallace, M.I. High-Throughput Optical Sensing of Nucleic Acids in a Nanopore Array. Nat. Nanotechnol. 2015, 10, 986-991. [CrossRef] [PubMed]

12. McNally, B.; Singer, A.; Yu, Z.; Sun, Y.; Weng, Z.; Meller, A. Optical Recognition of Converted DNA Nucleotides for Single-Molecule DNA Sequencing Using Nanopore Arrays. Nano Lett. 2010, 10, 2237-2244. [CrossRef] [PubMed]

13. Bell, N.A.W.; Thacker, V.V.; Hernández-Ainsa, S.; Fuentes-Perez, M.E.; Moreno-Herrero, F.; Liedl, T.; Keyser, U.F. Multiplexed Ionic Current Sensing with Glass Nanopores. Lab Chip 2013, 13, 1859-1862. [CrossRef] [PubMed]

14. Vogel, R.; Willmott, G.; Kozak, D.; Roberts, G.S.; Anderson, W.; Groenewegen, L.; Glossop, B.; Barnett, A.; Turner, A.; Trau, M. Quantitative Sizing of Nano/Microparticles with a Tunable Elastomeric Pore Sensor. Anal. Chem. 2011, 83, 3499-3506. [CrossRef] [PubMed]

15. Liang, S.; Xiang, F.; Tang, Z.; Nouri, R.; He, X.; Dong, M.; Guan, W. Noise in Nanopore Sensors: Sources, Models, Reduction, and Benchmarking. Nanotechnol. Precis. Eng. 2020, 3, 9-17. [CrossRef]

16. He, Y.; Tsutsui, M.; Fan, C.; Taniguchi, M.; Kawai, T. Controlling DNA Translocation through Gate Modulation of Nanopore Wall Surface Charges. ACS Nano 2011, 5, 5509-5518. [CrossRef]

17. He, Y.; Tsutsui, M.; Fan, C.; Taniguchi, M.; Kawai, T. Gate Manipulation of DNA Capture into Nanopores. ACS Nano 2011, 5, 8391-8397. [CrossRef]

18. Golla, E. Use of Silicon as a Semiconductor. In Crystal Growth and Evaluation of Silicon for VLSI and ULSI; CRC Press: Boca Raton, FL, USA, 2014; p. 7.

19. Ristic, S.; Prijic, A.; Prijic, Z. Dependence of Static Dielectric Constant of Silicon on Resistivity at Room Temperature. Serb. J. Electr. Eng. 2004, 1, 237-247. [CrossRef]

20. Dow, H.S.; Kim, W.S.; Lee, J.W. Thermal and Electrical Properties of Silicon Nitride Substrates. AIP Adv. 2017, 7, 095022. [CrossRef]

21. Chou, C.Y.; Lin, C.H.; Chen, W.H.; Li, B.J.; Liu, C.Y. High-Dielectric-Constant Silicon Nitride Thin Films Fabricated by Radio Frequency Sputtering in Ar and Ar/N2 Gas Mixture. Thin Solid Films 2020, 709, 138198. [CrossRef]

22. Masetti, G.; Severi, M.; Solmi, S. Modeling of Carrier Mobility Against Carrier Concentration in Arsenic-, Phosphorus-, and Boron-Doped Silicon. IEEE Trans. Electron. Dev. 1983, 30, 764-765. [CrossRef]

23. Zeng, S.; Wen, C.; Solomon, P.; Zhang, S.L.; Zhang, Z. Rectification of Protein Translocation in Truncated Pyramidal Nanopores. Nat. Nanotechnol. 2019, 14, 1056-1062. [CrossRef] [PubMed]

24. Tsutsui, M.; Maeda, Y.; He, Y.; Hongo, S.; Ryuzaki, S.; Kawano, S.; Kawai, T.; Taniguchi, M. Trapping and Identifying Single-Nanoparticles Using a Low-Aspect-Ratio Nanopore. Appl. Phys. Lett. 2013, 103, 013108. [CrossRef]

25. Laohakunakorn, N.; Thacker, V.; Muthukumar, M.; Keyser, U. Electroosmotic Flow Reversal outside Glass Nanopores. Nano Lett. 2015, 15, 695-702. [CrossRef]

26. Arjmandi, N.; Van Roy, W.; Lagae, L.; Borghs, G. Measuring the Electric Charge and Zeta Potential of Nanometer-Sized Objects Using Pyramidal-Shaped Nanopores. Anal. Chem. 2012, 84, 8490-8496. [CrossRef]

27. Langecker, M.; Pedone, D.; Simmel, F.C.; Rant, U. Electrophoretic Time-of-Flight Measurements of Single DNA Molecules with Two Stacked Nanopores. Nano Lett. 2011, 11, 5002-5007. [CrossRef]

28. Matsuda, R.; Ryuzaki, S.; Okamoto, K.; Arima, Y.; Tsutsui, M.; Taniguchi, M.; Tamada, K. Finite-Difference Time-Domain Simulations of Inverted Cone-Shaped Plasmonic Nanopore Structures. J. Appl. Phys. 2020, 127, 243109. [CrossRef]

29. Davenport, M.; Healy, K.; Pevarnik, M.; Teslich, N.; Cabrini, S.; Morrison, A.P.; Siwy, Z.S.; Létant, S.E. The Role of Pore Geometry in Single Nanoparticle Detection. ACS Nano 2012, 6, 8366-8380. [CrossRef]

(C) 2020 by the authors. Licensee MDPI, Basel, Switzerland. This article is an open access article distributed under the terms and conditions of the Creative Commons Attribution (CC BY) license (http://creativecommons.org/licenses/by/4.0/). 